\title{
Nanocellulose Based Sustainable Dyeing of Cotton Textiles with Minimized Water Pollution
}

Anuradhi Liyanapathiranage ${ }^{\dagger}$, Maria J. Peña ${ }^{\ddagger}$, Suraj Sharma ${ }^{\dagger *}$, and Sergiy Minko ${ }^{\dagger *}$

${ }^{\dagger}$ Nanostructured Materials Lab, University of Georgia, Athens, GA 30602. USA

${ }^{\ddagger}$ Complex Carbohydrate Research Center, University of Georgia, Athens, GA 30602. USA

\section{Supporting Information}

NFC dyeing

Table S1. Typical formulation for dyeing of NFC hydrogel.

\begin{tabular}{r|c|} 
Material & Amounts used $(g)$ \\
\cline { 2 - 2 } 2 wt.\% NFC gel & 100.0 \\
\cline { 2 - 2 } Reactive dye (s) & 0.50 \\
\cline { 2 - 2 } $\mathrm{Na}_{2} \mathrm{SO}_{4}(\mathrm{~s})$ & 3.5 \\
\cline { 2 - 2 } $\mathrm{Na}_{2} \mathrm{CO}_{3}(\mathrm{~s})$ & 0.64 \\
\cline { 2 - 2 } Water & 60.0 \\
&
\end{tabular}

Table S2. NFC dyeing formulations with different liquor ratios, LR (water to NFC weight ratio).

\begin{tabular}{|c|c|c|c|r|}
\cline { 2 - 5 } Material & $L R 80$ & $L R 100$ & LR 120 & LR 140 \\
\cline { 2 - 5 } & \multicolumn{4}{|c|}{ Loaded Amount, $g$} \\
\cline { 2 - 5 } 2 wt.\% NFC gel & 100 & 100 & 100 & 100 \\
\cline { 2 - 5 } Reactive dye (s) & 0.51 & 0.63 & 0.75 & 0.88 \\
\cline { 2 - 5 } $\mathrm{Na}_{2} \mathrm{SO}_{4}(\mathrm{~s})$ & 2.52 & 3.16 & 3.84 & 4.5 \\
\cline { 2 - 5 } $\mathrm{Na}_{2} \mathrm{CO}_{3}(\mathrm{~s})$ & 0.64 & 0.80 & 0.96 & 1.12 \\
\cline { 2 - 5 } Water (1) & 60 & 100 & 140 & 180 \\
\cline { 2 - 5 } & & &
\end{tabular}

Table S3. Formulation for conjugation of soluble polysugars with reactive dyes.

\begin{tabular}{|l|r|r|}
\hline & $\begin{array}{l}\text { Soluble } \\
\text { Fraction from } \\
(2 \text { wt.\% NFC })\end{array}$ & Exhaust Dye Bath \\
\hline Water, g & 60 & 60 \\
\hline Dye, $\mathrm{g}$ & 0.21 & 0.21 \\
\hline Sodium Sulphate (320 g/L), g & 3.84 & 3.84 \\
\hline Sodium Carbonate, $\mathrm{g}$ & 0.20 & 0.20 \\
\hline
\end{tabular}




\section{Textile dyeing and post-treatment}

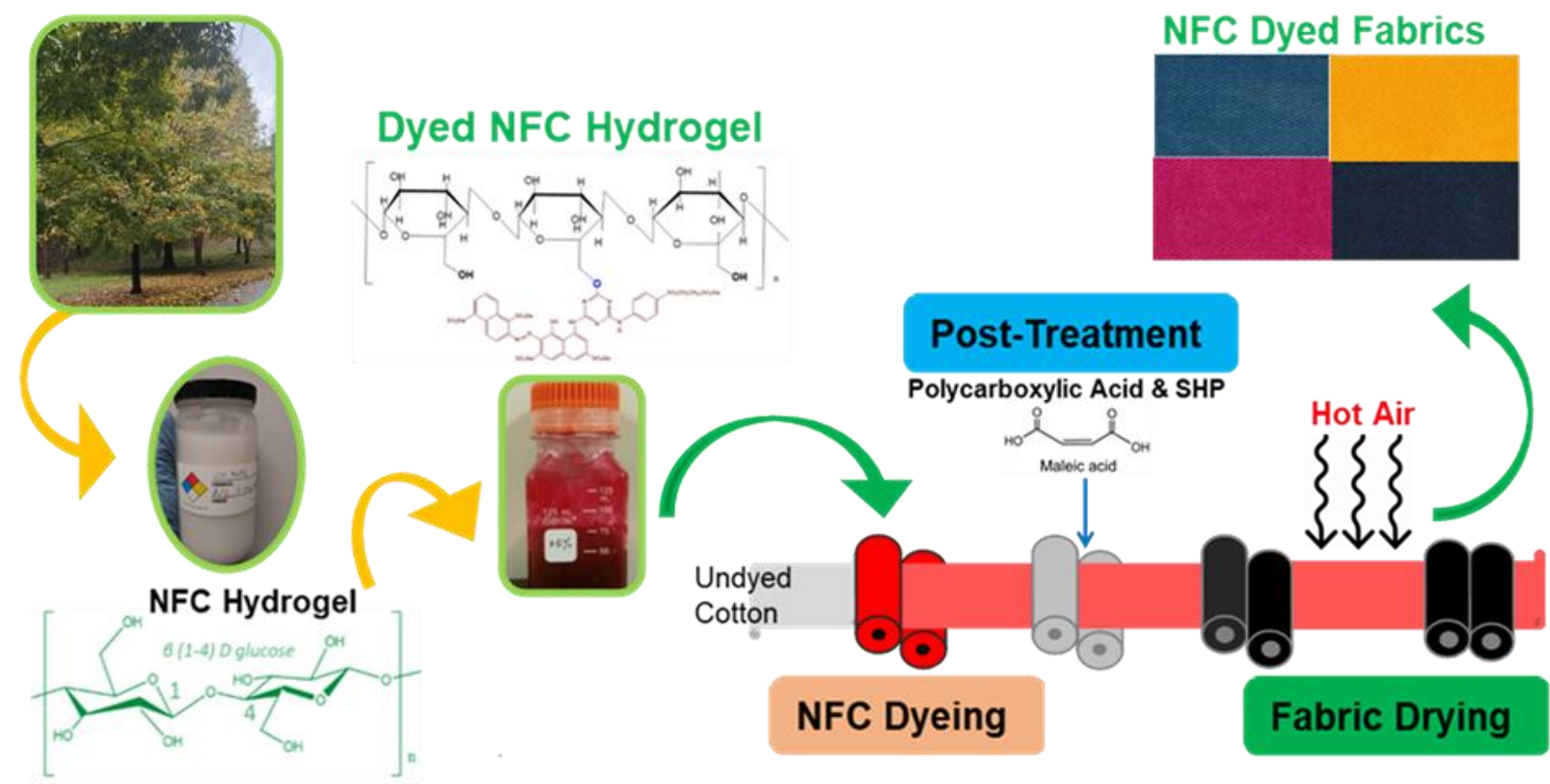

Figure S1. Schematic of NFC dyeing and post-treatment: NFC hydrogel is colored with a reactive dye yielding the NFC-dye pigment via covalent binding of the reactive dye with NFC fibrils. The colored hydrogel is applied to the cotton fabrics, then post-treated with polycarboxylic acids, and dried at elevated temperatures to form crosslinks between NFC-dye pigment and cotton.

(I)
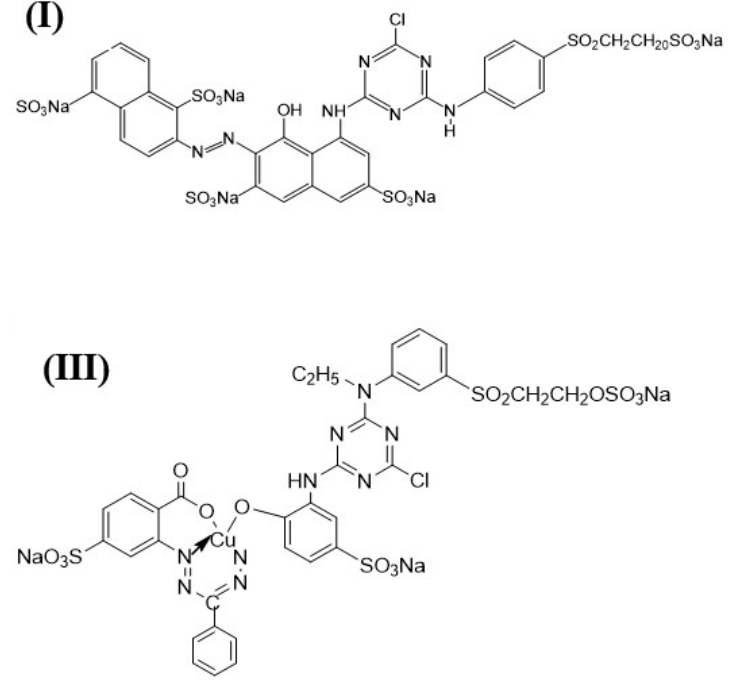

(II)
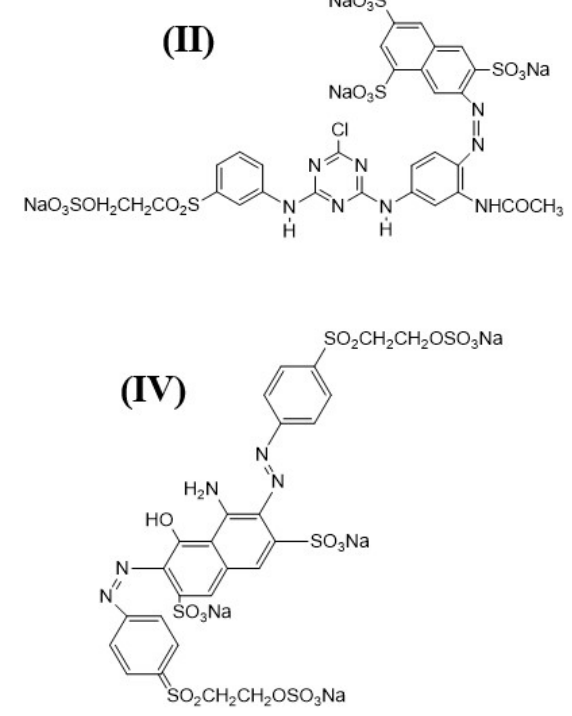

Figure S2. Chemical structure of reactive dyes: I- Reactive Red -Everzol Red 3BS, II- Reactive Yellow- Everzol Yellow 3RS, III- Reactive Blue- Everzol BRF and IV- Reactive Black-Everzol BHC 


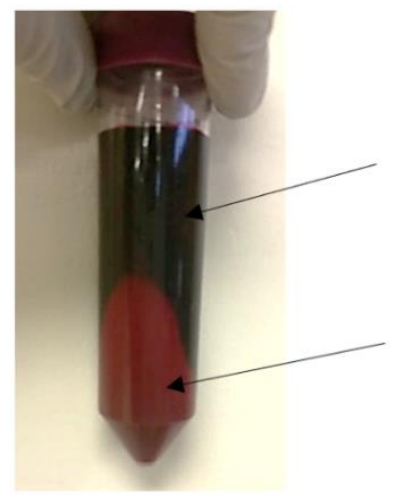

a

NFC-Dye supernatant

NFC-Dye bound fraction

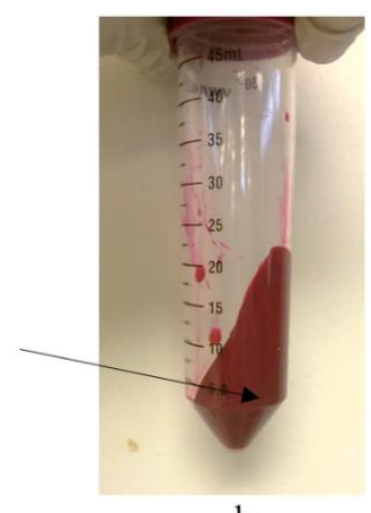

$\mathrm{b}$

Figure S3. A sample of NFC-dye hydrogel (a) after centrifugation and (b) followed by removal of the supernatant. The results demonstrates that the some amount of the reactive dye remains in solution and contribute to pollutions of the wastewater.

\section{Analysis of soluble polysugars.}

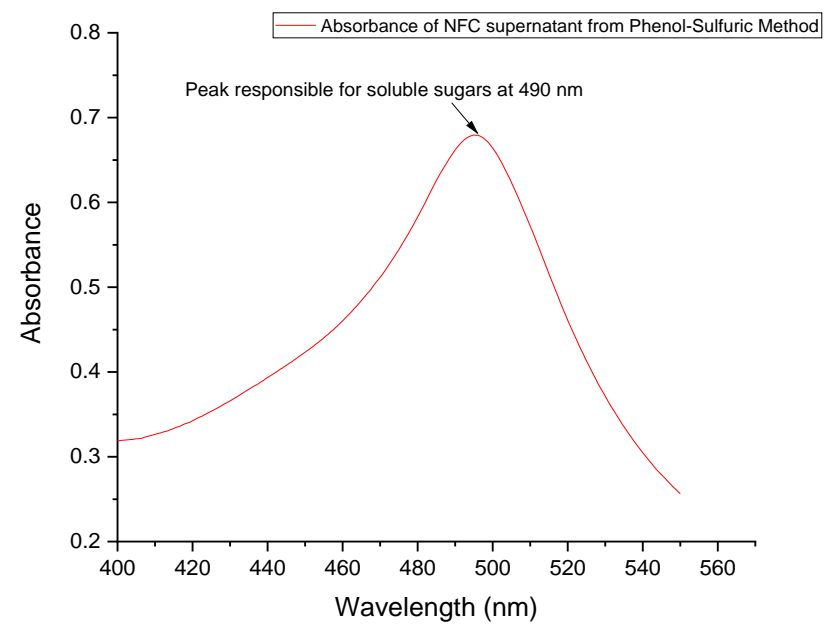

Figure S4. UV-vis absorption spectrum (phenol-sulfuric acid method) of the supernatant of the 2 wt.\% NFC hydrogel (without CMC) at $490 \mathrm{~nm}$ indicates the presence of carbohydrates. (in presence of sulfuric acids, simple sugars and polysugars react with phenol to yield yellow-gold colored mixture). 


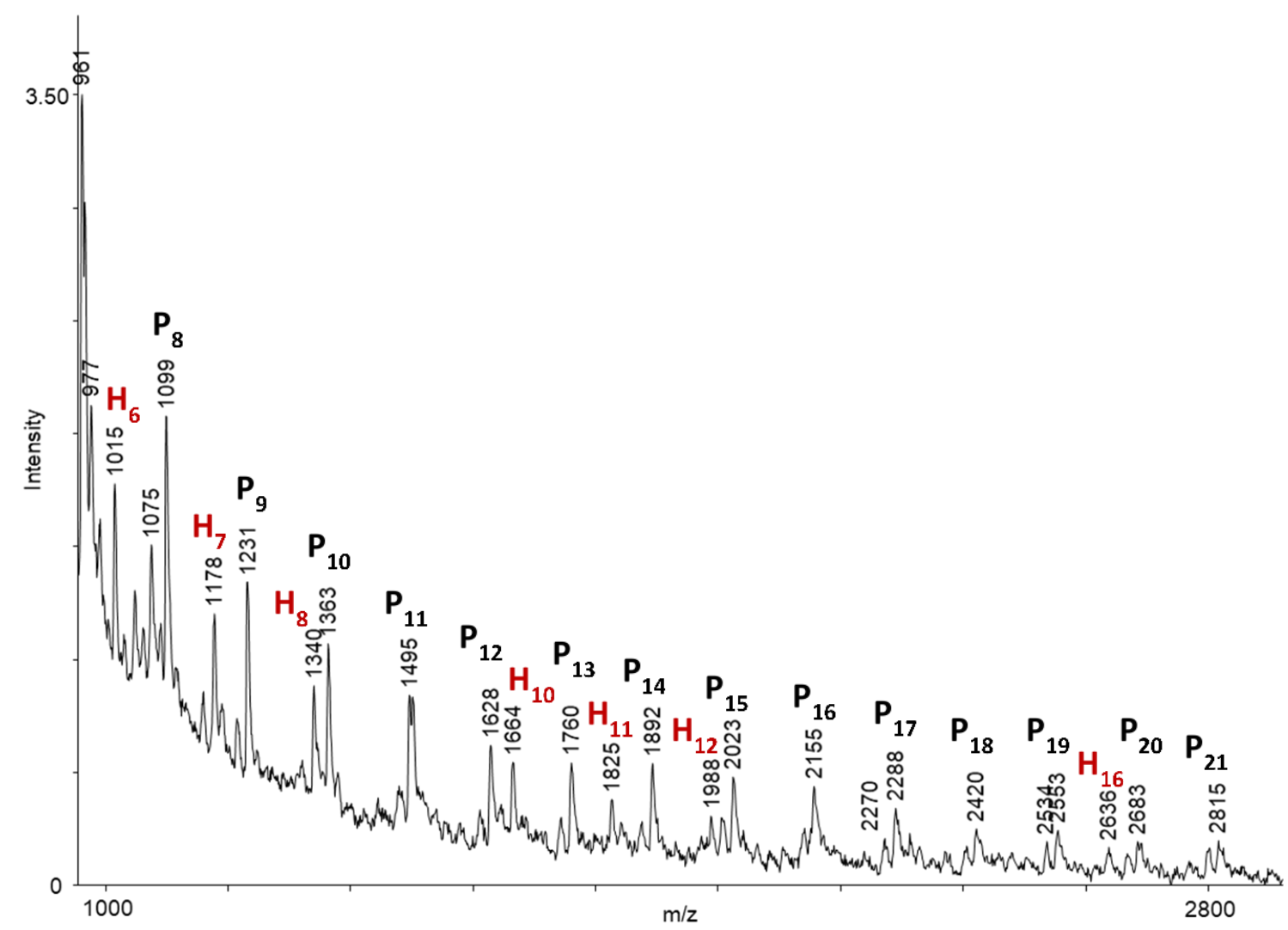

Figure S5. MALDI-TOF mass spectrum of the oligosaccharides presence in the supernatant of 2 wt.\% NFC hydrogel. All the ions are $[\mathrm{M}+\mathrm{Na}]$ adducts. The labels correspond to oligosaccharides containing pentoses $(\mathrm{P})$ and hexoses $(\mathrm{H})$. The subscript number indicate the degree of polymeration of the oligosaccharide. 


\section{Cross-linking of NFC-dye upon post-treatment with polycarboxylic acids}

NFC-Dye fibrous pigment matrix

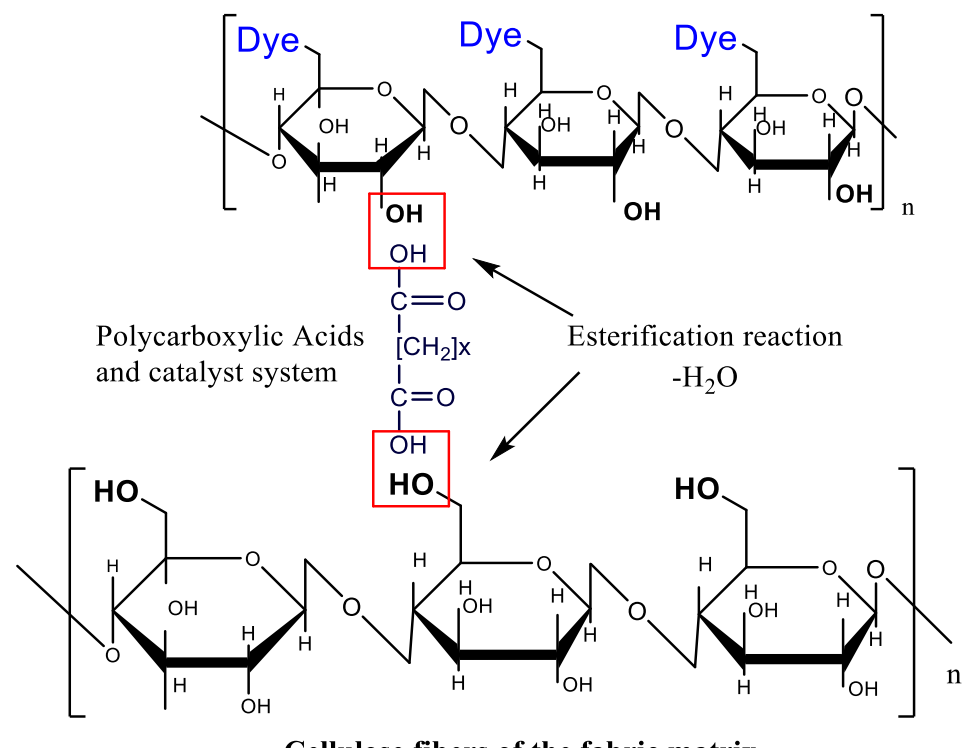

Figure S6. Schematic of the cross-linking of NFC-dyes with polyacrboxylic acids via esterification reaction.

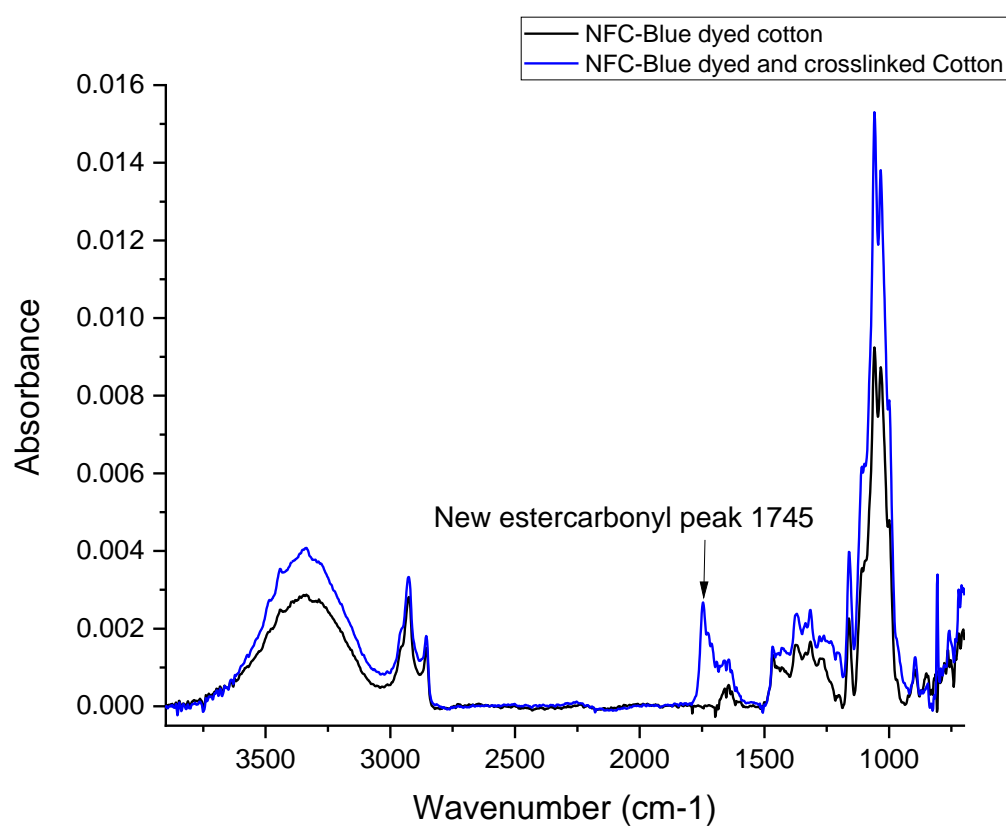

Figure S7. FTIR-spectra characterization of crosslinks between NFC-Reactive Blue dye and cellulose fibers 


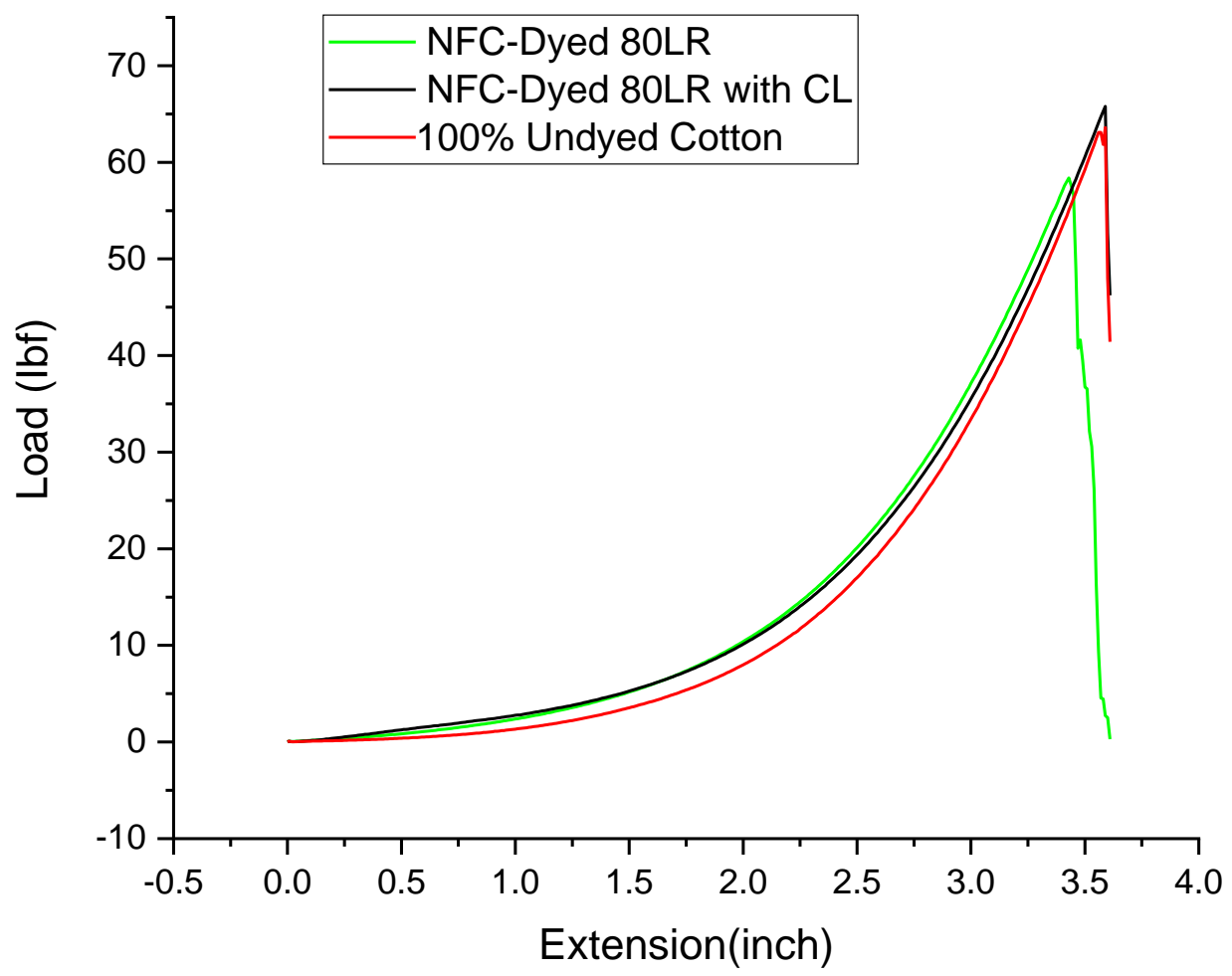

Figure S8. Tensile test data of 100\% undyed cotton, NFC-dyed cotton (at 80 LR), and NFCdyed cotton underwent PCA post-treament<smiles>O=C(O)C=CC(=O)O</smiles>

(V)<smiles>C=C(CC(=O)O)C(=O)O</smiles>

(VI)<smiles>O=C(O)CC(O)(CC(=O)O)C(=O)O</smiles>

(VII)

Figure S9. Chemical structure of polycarboxylic acids: V - maleic acid, VI -. itaconic acid, and VI - citric acid 